\title{
VADD: Vehicle-Assisted Data Delivery in Vehicular Ad Hoc Networks
}

\author{
Jing Zhao, Student Member, IEEE, and Guohong Cao, Senior Member, IEEE
}

\begin{abstract}
Multihop data delivery through vehicular ad hoc networks is complicated by the fact that vehicular networks are highly mobile and frequently disconnected. To address this issue, we adopt the idea of carry and forward, where a moving vehicle carries a packet until a new vehicle moves into its vicinity and forwards the packet. Being different from existing carry and forward solutions, we make use of predictable vehicle mobility, which is limited by traffic pattern and road layout. Based on the existing traffic pattern, a vehicle can find the next road to forward the packet to reduce the delay. We propose several vehicle-assisted data delivery (VADD) protocols to forward the packet to the best road with the lowest data-delivery delay. Experimental results show that the proposed VADD protocols outperform existing solutions in terms of packet-delivery ratio, data packet delay, and protocol overhead. Among the proposed VADD protocols, the Hybrid Probe (H-VADD) protocol has a much better performance.
\end{abstract}

Index Terms - Carry and forward, data delivery, routing, vehicular networks, wireless networks.

\section{INTRODUCTION}

$\mathbf{V}$ EHICULAR ad hoc networks (VANETs) have been envisioned to be useful in road safety and many commercial applications [1], [2]. For example, a vehicular network can be used to alert drivers to potential traffic jams, providing increased convenience and efficiency. It can also be used to propagate emergency warning to drivers behind a vehicle (or incident) to avoid multicar collisions. To realize this vision, FCC has allocated $75 \mathrm{MHz}$ of spectrum for dedicated shortrange communications (vehicle-vehicle or vehicle-roadside), and IEEE is working on standard specifications for intervehicle communication. As more and more vehicles are equipped with communication capabilities that allow intervehicle communication, large-scale VANETs are expected to be available in the near future.

Quite a bit of research has been done on intervehicle communication. Medium access control (MAC) issues have been addressed in [1], [3], and [4], where slot-reservation MAC protocols [3], [4] and congestion control policies for emergency warning [1] are studied. Transportation safety issues have been

Manuscript received April 21, 2006; revised September 13, 2006, February 16, 2007, and March 7, 2007. This work was supported in part by the Army Research Office under Grant W911NF-05-1-0270 and by the National Science Foundation under Grant CNS-0092770, Grant CNS-0519460, and Grant CNS-0721479. A preliminary version [23] of this paper appeared in Infocom'06. The review of this paper was coordinated by Prof. J. Li.

The authors are with the Department of Computer Science and Engineering, The Pennsylvania State University, University Park, PA 16802 USA (e-mail: jizhao@cse.psu.edu; gcao@cse.psu.edu).

Color versions of one or more of the figures in this paper are available online at http://ieeexplore.iee.org.

Digital Object Identifier 10.1109/TVT.2007.901869 addressed in [2] and [5], where vehicles communicate with each other and with the static network nodes such as traffic lights, bus shelters, and traffic cameras. Data dissemination protocols [6], [7] have been proposed to disseminate information about traffic, obstacles, and hazards on the roads. Other applications, such as real-time video streaming between vehicles, have been studied in [8].

Most of the aforementioned works are limited to one-hop or short-range multihop communication. On the other hand, VANETs are also useful to other scenarios. For example, without Internet connection, a moving vehicle may want to query a data center several miles away through a VANET. To further motivate our work, consider the widely deployed wireless LANs or infostations [9], [10], which can be used to deliver advertisements and announcements such as sale information or remaining stock at a department store, available parking in a parking lot, and the meeting schedule at a conference room. Since the broadcast range is limited, only clients around the access point can directly receive the data. However, these data may be beneficial for people in moving vehicles which are far away. For example, people driving who wants to shop may want to query several department stores to decide where to go; a driver may query the traffic cameras or parking lot information to make a better road plan. All these queries may be issued miles or tens of miles away from the broadcast site. With a VANET, the requester can send the query to the broadcast site and get a reply from it. In the aforementioned applications, the users can tolerate up to seconds or minute of delay as long as the reply will eventually return.

Although the aforementioned services can be supported by a wireless infrastructure (e.g., 3G), the cost of doing this is high and may not be possible when such an infrastructure does not exist or is damaged. From the service provider's point of view, setting up a wireless LAN is very cheap, but the cost of connecting it to the Internet or the wireless infrastructure is high. From the user point of view, the cost of accessing data through a wireless carrier is still high, and most of the cellular phone users are limited to voice service. Moreover, in case of disaster, the wireless infrastructure may be damaged, whereas wireless LANs and vehicular networks can be used to provide important traffic, rescue, and evacuation information to the users.

Multihop data delivery through VANETs is complicated by the fact that vehicular networks are highly mobile and sometimes sparse. Network density is related to traffic density, which is affected by location and time. For example, the traffic density is low in rural areas and during night but very high in the large populated area and during rush hours. Although it is 
very difficult to find an end-to-end connection for a sparsely connected network, the high mobility of vehicular networks introduces opportunities for mobile vehicles to intermittently connect with each other when moving. Namboodiri et al. [11] showed that there is a high chance for moving vehicles to set up a short path with a few hops in a highway model. Furthermore, a moving vehicle can carry the packet and forward it to the next vehicle. Through relays and carry and forward, the message can be delivered to the destination without an end-toend connection for delay-tolerant applications.

This paper studies the problem of efficient data delivery in VANETs. Specifically, when a vehicle issues a delay-tolerant data query to some fixed site, we propose techniques to efficiently route the packet to that site and receive the reply within a reasonable delay. The proposed vehicle-assisted data delivery (VADD) is based on the idea of carry and forward [12], where nodes carry the packet when routes do not exist and forward the packet to the new receiver that moves into its vicinity. Being different from existing carry and forwarding approaches [12]-[14], it makes use of the predictable mobility in a VANET, which is limited by traffic pattern and road layout. Extensive experiments are used to evaluate the proposed data-delivery protocols. Results show that the proposed VADD protocols outperform existing solutions in terms of packet-delivery ratio, data packet delay, and protocol overhead.

The rest of this paper is organized as follows. Section II describes how to model the data-delivery delay. The VADD protocols will be presented in Section III. Section IV evaluates the performance of the proposed protocols. Section V concludes this paper.

\section{VADD MODEL}

In this section, we first give the assumptions and the overview of VADD and then present the VADD delay model.

\section{A. Assumptions}

We assume that vehicles communicate with each other through a short-range wireless channel (100-250 m). The packet-delivery information, such as source ID, source location, packet generation time, destination location, expiration time, etc., is specified by the data source and is placed in the packet header. A vehicle knows its location by triangulation or through a GPS device, which is already popular in new cars and will be common in the future. Vehicles enclose their own physical location, moving velocity, and direction information in their periodic beacon messages, and this information can be overheard by their one-hop neighbors.

We assume that vehicles are equipped with preloaded digital maps, which provide street-level map and traffic statistics such as traffic density, vehicle speed on roads at different times of the day, and traffic signal schedule (e.g., the length of red signal interval) at intersections. Such kind of digital map has already been commercialized. The latest one is developed by MapMechanics [15], which includes road speed data and an indication of the relative density of vehicles on each road. Yahoo is also working on integrating traffic statistics in its

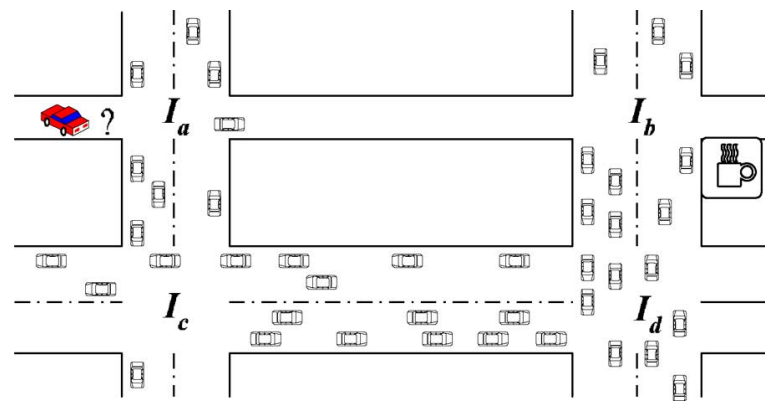

Fig. 1. Find a path to the coffee shop.

new version of Yahoo Maps, where real traffic reports of major U.S. cities are available. We expect that more detailed traffic statistics will be integrated into digital map in the near future. Note that the cost of setting up such a vehicular network can be justified by its application to many road safety and commercial applications [1], [2], [5], which are not limited to the proposed delay-tolerant data-delivery applications.

\section{B. VADD Overview}

VADD is based on the idea of carry and forward. The most important issue is to select a forwarding path with the smallest packet-delivery delay. Although geographical forwarding approaches such as greedy perimeter stateless routing (GPSR) [16], which always chooses the next hop closer to the destination, are very efficient for data delivery in $a d$ hoc networks, they may not be suitable for sparsely connected vehicular networks.

As shown in Fig. 1, suppose that a driver approaches intersection $I_{a}$ and sends a request to the coffee shop (to make a reservation) at the corner of intersection $I_{b}$. To forward the request through $I_{a} \rightarrow I_{c}, I_{c} \rightarrow I_{d}$, and $I_{d} \rightarrow I_{b}$ would be faster than through $I_{a} \rightarrow I_{b}$, even though the latter geographically provides the shortest possible path. The reason is that, in case of disconnection, the packet has to be carried by the vehicle, whose moving speed is significantly slower than wireless communication.

In sparsely connected networks, vehicles should try to make use of the wireless communication channel or, otherwise, resort to vehicles that are moving faster. Thus, our VADD follows the following basic principles.

1) Transmit through wireless channels, as much as possible.

2) If the packet has to be carried through certain roads, the road with higher speed should be chosen.

3) Due to the unpredictable nature of VANETs, we cannot expect the packet to be successfully routed along the precomputed optimal path, so dynamic path selection should continuously be executed throughout the packetforwarding process.

As shown in Fig. 2, VADD has three packet modes, namely, Intersection, StraightWay, and Destination, based on the location of the packet carrier (i.e., the vehicle that carries the packet). By switching between these packet modes, the packet carrier takes the best packet-forwarding path. Among the three modes, the Intersection Mode is the most critical and complicated one since vehicles have more choices at the intersection. 


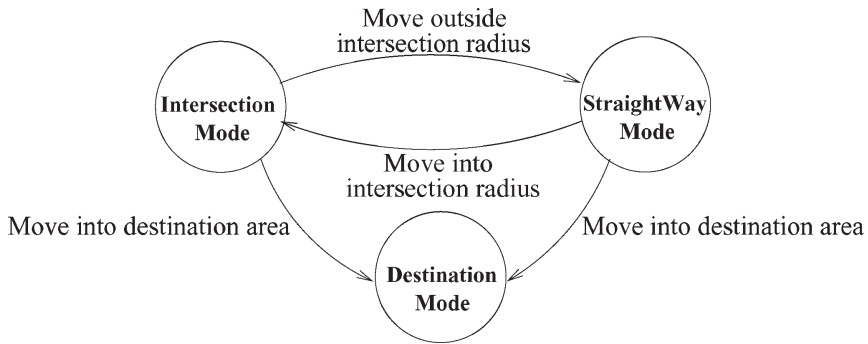

Fig. 2. Transition modes in VADD.

\section{VADD Delay Model}

To formally define the packet-delivery delay, we need the following notations.

1) $r_{i j}$ : the road from $I_{i}$ to $I_{j}$;

2) $l_{i j}$ : the Euclidean distance of $r_{i j}$;

3) $\rho_{i j}$ : the vehicle density on $r_{i j}$;

4) $v_{i j}$ : the average vehicle velocity on $r_{i j}$;

5) $d_{i j}$ : the expected packet-forwarding delay from $I_{i}$ to $I_{j}$.

We assume that the intervehicle distances follow an exponential distribution, with a mean distance equal to $1 / \rho_{i j}$. Thus

$$
d_{i j}=\left(1-e^{-R \cdot \rho_{i j}}\right) \cdot \frac{l_{i j} \cdot c}{R}+e^{-R \cdot \rho_{i j}} \cdot \frac{l_{i j}}{v_{i j}}
$$

where $R$ is the wireless transmission range, and $c$ is the average one-hop packet transmission delay. Equation (1) indicates that the intervehicle distances are smaller than $R$ on a portion of $1-e^{-R / \rho_{i j}}$ of the road, where wireless transmission is used to forward the packet. On the rest of the road, vehicles are used to carry the data. Apparently, a larger traffic density makes up a small portion completed by vehicle motion.

One way of viewing the VADD delay model is to represent the vehicular network with a directed graph, in which nodes represent intersections and edges represent the roads connecting adjacent intersections. The direction of each edge is the traffic direction. The packet-forwarding delay between two adjacent intersections is the weight of the edge. Given the weight on each edge, a naive optimal forwarding path selection scheme is to compute the shortest path from source to destination by applying Dijkstra's algorithm. However, this simple solution does not work since we cannot freely select the outgoing edge to forward the packet at an intersection. Only those edges with vehicles on it that will be used to carry packets can be the candidate path for packet forwarding. However, we do not know, for sure, which direction the packet will go at the next intersection. In other words, it is impossible to compute the complete packet-forwarding path.

To address this problem, we propose a stochastic model to estimate the data-delivery delay, which is used to select the next road (intersection). We first introduce the following notations:

1) $D_{i j}$ : the expected packet-delivery delay from $I_{i}$ to the destination if the packet carrier at $I_{i}$ chooses to deliver the packet following road $r_{i j}$;

2) $P_{i j}$ : the probability that the packet is forwarded through road $r_{i j}$ at $I_{i}$

3) $N(j)$ : the set of neighboring intersections of $I_{j}$.

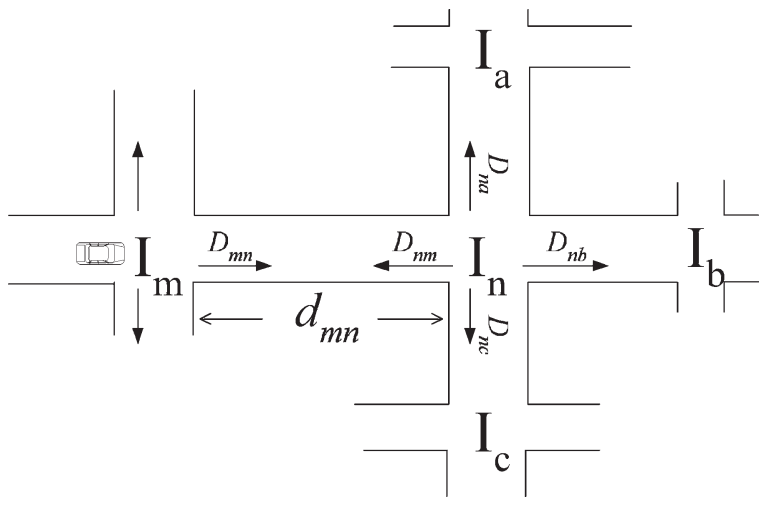

Fig. 3. Example of the VADD delay model.

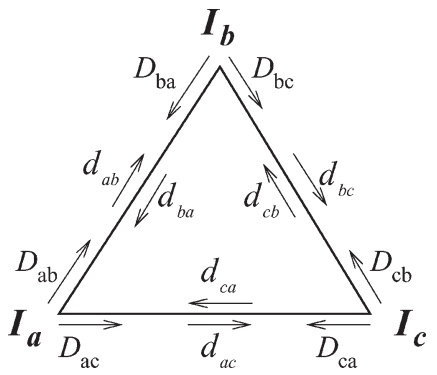

Fig. 4. One road graph.

As shown in Fig. 3, for a packet at $I_{m}$, the expected delay of delivering the packet through road $r_{m n}$ is

$$
D_{m n}=d_{m n}+\sum_{j \in N(n)}\left(P_{n j} \times D_{n j}\right) .
$$

Fig. 4 shows how to apply (2) to a simple triangle road which only contains three intersections $I_{a}, I_{b}$, and $I_{c}$. Suppose that a data packet reaches $I_{a}$, and the destination is $I_{c}$. The forwarding scheme needs to decide whether to forward the packet through the road to $I_{c}$ or $I_{b}$. This is done by computing the value of $D_{a c}$ and $D_{a b}$ and by choosing the smaller one. By applying (2), we have the following linear equations:

$$
\left\{\begin{array}{l}
D_{a c}=d_{a c} \\
D_{a b}=d_{a b}+P_{b a} \cdot D_{b a}+P_{b c} \cdot D_{b c} \\
D_{b a}=d_{b a}+P_{a b} \cdot D_{a b}+P_{a c} \cdot D_{a c} \\
D_{b c}=d_{b c} \\
D_{c b}=0 \\
D_{c a}=0 .
\end{array}\right.
$$

Note that both $d_{c b}$ and $d_{c a}$ are equal to zero since the packet already arrives at destination $I_{c}$, and they will not be forwarded anymore. We can easily solve (3) and get $D_{a c}$ and $D_{a b}$

$$
\begin{aligned}
D_{a c}= & d_{a c} \\
D_{a b}= & \frac{1}{1-P_{a b} \cdot P_{b a}} \\
& \times\left(d_{a b}+P_{b a} \cdot d_{b a}+P_{b a} \cdot P_{a c} \cdot d_{a c}+P_{b c} \cdot d_{b c}\right) .
\end{aligned}
$$

Unfortunately, finding the minimum forwarding delay between two arbitrary intersections is impossible since it involves 


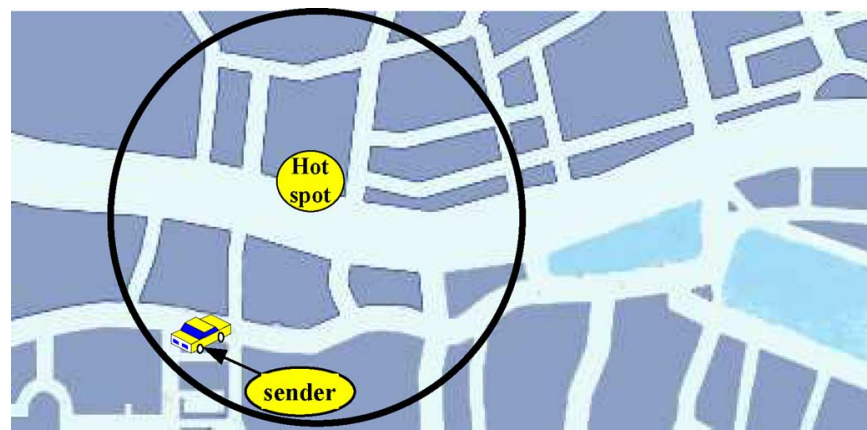

Fig. 5. Add a boundary.

unlimited unknown intersections. However, by placing a boundary, including the source and the destination in a connected graph, we are able to find the expected minimum forwarding delay between them. Fig. 5 shows one such boundary which includes the sender and the destination (hot spot). The boundary used in this paper is a circle, with its center point at the destination. The radius of the boundary circle is $4000 \mathrm{~m}$ if the distance between the packet and the destination is less than $3000 \mathrm{~m}$; otherwise, the radius is the distance between the packet and the destination plus $1000 \mathrm{~m}$. Certainly, there are many other ways to place the boundary as long as the destination is enclosed. Since only the roads within the boundary are used as available paths to compute the delay, a large boundary covering more high-density streets can generally find more close-tooptimal paths but with more computation overhead. Thus, there is a tradeoff between computational complexity and accuracy in delay estimation when selecting the boundary. Since this is not the major concern of this paper and it does not affect the correctness of our algorithms, we will not further discuss it in this paper.

Since the number of intersections inside the boundary is finite, we can derive (2) for each outgoing edge of every intersection within the boundary [similar to the method used to derive (3)]. In this way, an $n \times n$ linear equation system is generated, where $n$ is the number of roads within the boundary.

To follow the general representation of linear equation systems, we rename the unknown $D_{i j}$ as $x_{i j}$, rename the subscript $i j$ of $d_{i j}$ and $x_{i j}$ with a unique number for each pair $i j$, and rename the subscript of $P_{i j}$ by its position in the equations. Then, we can derive $n$ linear equations with $n$ unknowns $x_{1}, x_{2}, \ldots, x_{n}$

$$
\begin{aligned}
x_{1}= & d_{1}+P_{11} x_{1}+P_{12} x_{2}+\cdots+P_{1 n} x_{n} \\
x_{2}= & d_{2}+P_{21} x_{1}+P_{22} x_{2}+\cdots+P_{2 n} x_{n} \\
& \vdots \\
x_{n}= & d_{n}+P_{n 1} x_{1}+P_{n 2} x_{2}+\cdots+P_{n n} x_{n} .
\end{aligned}
$$

It can easily be transformed to the following matrix:

$$
\begin{array}{ccc}
\left(P_{11}-1\right) x_{1}+P_{12} x_{2} & +\cdots+P_{1 n} x_{n} & =-d_{1} \\
P_{21} x_{1}+\left(P_{22}-1\right) x_{2} & +\cdots+P_{2 n} x_{n} & =-d_{2} \\
& \vdots & \\
P_{n 1} x_{1}+P_{n 2} x_{2} & +\cdots+\left(P_{n n}-1\right) x_{n} & =-d_{n}
\end{array}
$$

which is equivalent to

$$
(P-E) \cdot X=-D
$$

where

$$
\begin{aligned}
P & =\left[\begin{array}{cccc}
P_{11} & P_{12} & \cdots & P_{1 n} \\
P_{21} & P_{22} & \cdots & P_{2 n} \\
\vdots & \vdots & \ddots & \vdots \\
P_{n 1} & P_{n 2} & \cdots & P_{n n}
\end{array}\right] \\
E & =\left[\begin{array}{cccc}
1 & 0 & \cdots & 0 \\
0 & 1 & \cdots & 0 \\
\vdots & \vdots & \ddots & \vdots \\
0 & 0 & \cdots & 1
\end{array}\right] \\
X & =\left[\begin{array}{c}
x_{1} \\
x_{2} \\
\vdots \\
x_{n}
\end{array}\right] \text { and } D=\left[\begin{array}{c}
d_{1} \\
d_{2} \\
\vdots \\
d_{n}
\end{array}\right] .
\end{aligned}
$$

We can prove that this linear equation system has one unique solution (see the Appendix). The typical way to solve this equation is to use the Gaussian elimination algorithm, which is solved in time $\Theta\left(n^{3}\right)$.

By solving (4), we get $D_{i j}$ for the current intersection $I_{i}$. The packet carrier can sort $D_{i j}$ for each neighboring intersection $I_{j}$ and forward the packet to the road with smaller delay. As a result, among all the vehicles within a communication range (called contacts) available at the intersection, the packet will be forwarded to the one on the road with the smallest delay. If no contact is available or all available contacts are going through roads with longer delay than the packet carrier's next traveling road, the packet carrier passes the intersection with the packet and looks for the next forwarding opportunity.

\section{VADD PROTOCOLS}

In this section, we present the VADD protocols. We first present the protocols used in the Intersection Mode and then present the contact model and protocols on the Straightway.

\section{A. VADD Protocols Used in the Intersection Mode}

By deriving and solving (4) at the intersection, the packet carrier can sort all the outgoing directions and check if there is a contact available to help forward through that direction. However, to determine the next hop among all available contacts and ensure a packet to go through the precomputed direction is not trivial. As shown in Fig. 6, vehicle $A$ has a packet to forward to certain destination. Assume that the optimal direction for this packet is North. There are two available contacts for the packet carrier: $B$ moving South and $C$ moving North. $A$ has two choices on selecting the next hop for the packet: $B$ or $C$. Both choices aim at forwarding the packet toward the North: selecting $B$ because $B$ is geographically closer to the North and provides a better possibility to exploit wireless communication (e.g., $B$ can immediately pass the packet to $D$, but $C$ cannot), or selecting $C$ because $C$ is moving in the packet-forwarding 


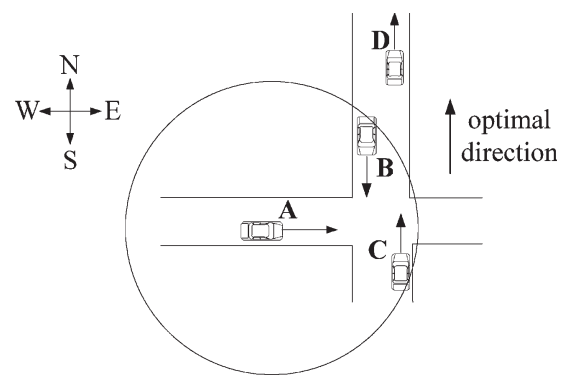

Fig. 6. Select the next vehicle to forward the packet.

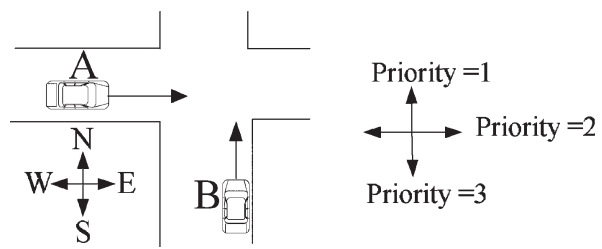

Fig. 7. Scenario of routing loop.

direction. These two choices lead to two different forwarding protocols: Location First Probe (L-VADD) and Direction First Probe (D-VADD).

1) Location First Probe (L-VADD): Given the preferred forwarding direction of a packet, L-VADD tries to find the closest contact toward that direction as the next hop. First, based on (4), $D_{i j}$ can be obtained for each outgoing road $r_{i j}$ at intersection $I_{i}$. As a result, each outgoing road is assigned a priority, where a smaller $D_{i j}$ has higher priority. Next, the packet carrier checks the outgoing directions starting from the highest priority. For a selected direction, the packet carrier chooses the next intersection toward the selected direction as the target intersection and applies geographical greedy forwarding toward the target intersection to pass the packet. If the current packet carrier cannot find any contact to the target intersection, it chooses the direction with the next lower priority and restarts the geographical greedy forwarding toward the new target intersection. This process continues until the selected direction has a lower priority than the packet carrier's current moving direction. At this time, the packet carrier will continue carrying the packet.

As shown in Fig. 6, vehicle $A$ forwards the packet to $B$. It seems like this is better than selecting $C$ as the next hop since $B$ can immediately forward the packet to $D$. Even if $D$ does not exist, selecting $B$ seems as good as selecting $C$ since $B$ will meet $C$ shortly and the packet can be passed to $C$ anyway. However, L-VADD may result in routing loops. Fig. 7 shows one of such a scenario. Assume that the North direction has the highest priority and the East has the second highest priority. $A$ first checks the North and cannot find any contact. Then, it checks the East and finds $B$, which is closer to the East. Thus, it forwards the packet to $B$. Upon receiving the packet, $B$ checks the North direction first, finds that $A$ is closer to the North, and then passes the packet back to $A$. There is a loop between $A$ and $B$.

A simple solution to break the routing loop is to record the previous hop(s) information. As in the above example, $A$ records its own ID as the previous_hop before forwarding the packet to $B$. When $B$ receives the packet and decides to forward the packet to $A$, it checks the previous hop record and finds that $A$ is the previous hop. To avoid a routing loop, $B$ will not forward the packet to $A$ and look for the next available contact.

A routing loop may involve $n(n>2)$ nodes. To detect such a routing loop, all these previous $n$ hops should be recorded. However, such loop detection mechanism dramatically degrades the forwarding performance since the detection mechanism may prevent many valid nodes from being considered as the next hop. As shown in Fig. 7, if $A$ is the packet carrier after a routing loop has been detected, there is no other contact available except $B$. Suppose that, after both $A$ and $B$ pass the center of the intersection, $A$ continues to go to the East and $B$ to the North. The packet should be forwarded to $B$ since $B$ will move toward the best direction, and the path between $A$ and $B$ becomes loop-free. However, as the packet records $B$ as the previous hop, forwarding the packet to $B$ is not allowed. Therefore, even though we can record the previous hop information to detect routing loops, many valid forwarding paths cannot be used.

2) Direction First Probe (D-VADD): Routing loop occurs because vehicles do not have a unanimous agreement on the order of the priority and, then, do not have an agreement on who should carry the packet. To address this issue, D-VADD ensures that everyone agrees on the priority order by letting the vehicle moving toward the desired packet-forwarding direction carry the packet.

In D-VADD, the direction selection process is the same as L-VADD. For a selected direction, instead of probing by location (in L-VADD), D-VADD selects the contacts moving toward the selected direction. Among the selected contacts, the one closest to the selected direction is chosen as the next hop. As shown in Fig. 6, D-VADD selects $C$ as the next hop when the selected direction is the North. Since $B$ is not moving North, it will not be considered. Therefore, D-VADD only probes vehicles moving toward the direction whose priority is higher than or equal to the moving direction of the current packet carrier. As the probing strictly follows the priority order of the direction, D-VADD has the following property: Any subsequent packet carrier moves toward the direction whose priority is higher than or equal to that of the current packet carrier.

Theorem 1: D-VADD is free from routing loops at intersection areas.

Proof: By contradiction, suppose that a routing loop occurs and node $A$ and $B$ are in the circle, which indicates that at least one packet forwarded from $A$ passes through $B$ and returns to $A$. Consider the first case where $A$ and $B$ are moving in the same direction, and the packet is forwarded from $A$ to $B$. It indicates that $B$ is closer to the destination direction than $A$, while the packet passing back to $A$ indicates the reverse. In the second case, if $A$ and $B$ move toward different directions, the packet forwarded from $A$ to $B$ indicates that $B$ is moving toward the direction of higher priority than $A \mathrm{~s}$, while the packet passing back to $A$ shows that $A$ 's direction has higher priority. Both cases lead to contradictions. Therefore, there is no routing loop in D-VADD.

3) Hybrid Probe (H-VADD): Compared to other VADD protocols, L-VADD without loop detection can minimize the packet-forwarding distance and, hence, the delay if there is no 
loop. However, the routing loop in L-VADD severely affects the performance and leads to a low packet-delivery ratio. Loop detection mechanism can remove the routing loop, but may also increase the forwarding delay. D-VADD is free from routing loops; however, they give priority to the moving direction and may suffer from long packet-forwarding distance and, hence, long packet-delivery delay.

An ideal VADD protocol should minimize the geographic forwarding distance and does not have routing loops. To achieve this goal, we design a scheme called H-VADD, which works as follows. Upon entering an intersection, H-VADD behaves like L-VADD with loop detection. If a routing loop is detected, it immediately uses D-VADD until it exits the current intersection. In this way, H-VADD inherits the advantage of using the shortest forwarding path in L-VADD when there is no routing loop and uses D-VADD to address the routing loop problem of L-VADD.

4) Problem of Disagreement and Redundant Computation: At an intersection, if the preferred forwarding direction of a packet is calculated at each hop of the forwarding nodes, the following two problems may occur.

Disagreement on preferred direction: Each node independently derives and solves (4) only based on the local information provided by their own digital maps. It is possible that two nodes do not exactly have the same traffic statistics (due to different map source, updating schedule, etc.). It is possible that two successive forwarding nodes obtain different expected forwarding delay for the same next road so that they may use different optimal directions to forward the packet. Then, the packet may suffer from routing loops, which is similar to that in L-VADD.

Redundant computation: In VADD, all the forwarding nodes within the same intersection area should exactly follow the same computation process and ideally get the same preferred forwarding direction for a given packet. Thus, it may waste computation resources if multiple nodes do the computation several times.

The aforementioned two problems exist in all three VADD protocols: L-VADD, D-VADD, and H-VADD. To deal with these problems, only the first node in the intersection area receiving the packet performs the computation and gets the priority order of the next forwarding direction/road for the packet. This information is enclosed in the packet header and kept until the packet is forwarded out of the current intersection. The subsequent forwarding nodes in the same intersection do not repeat the computation. Instead, they check the packet header and forward the packet based on the computed priority order. In this way, only one computation is performed for a packet at one intersection, and the disagreement problem will be solved.

\section{B. Calculating $P_{i j}$}

In this section, we provide solutions to calculate $P_{i j}$ used in Section II. Specifically, we choose D-VADD as the datadelivery protocol because of its simplicity in modeling the packet-forwarding process. Certainly, other protocols such as L-VADD and H-VADD can be modeled to calculate $P_{i j}$ in a similar way. The calculation of $P_{i j}$ under other VADD protocols should provide similar results since the different VADD protocols follow a similar principle to find the optimal forwarding path through the roads with high vehicle density.

We focus on the normal traffic layout, where each road has one- or two-way traffic and the intersections are either signalized or isolated [17]. Throughout this section, we assume that the vehicle arrivals at the intersections follow the Poisson distribution.

The expected time that a packet carrier stays in the Intersection Mode is referred to as the contacting time. The contacting time at a signalized intersection $I_{i}$, which is denoted as $t_{i}$, is only related to the length of the signal interval at $I_{i}$, and we assume that it can be obtained from the digital map. In an isolated intersection, vehicles in all directions can smoothly go through without being stopped. For a vehicle at $I_{i}$, we assume that the average vehicle speed in going through the intersection is the same as the average vehicle speed at the outgoing road. Let $R_{\text {int }}$ denote the radius of the intersection area, which is a circle area with the intersection point as the center. Equation (5) computes the contacting time $\left(T_{i j}\right)$ for packet carriers which enter intersection $I_{i}$ and move toward neighbor intersection $I_{j}$

$$
T_{i j}= \begin{cases}t_{i}, & I_{i} \text { is signalized } \\ \frac{2 R_{\text {int }}}{v_{i j}}, & I_{i} \text { is isolated. }\end{cases}
$$

The packet carrier is able to forward the packet toward road $r_{i j}$ at $I_{i}$ only if it can meet at least one contact going toward road $r_{i j}$. Next, we calculate the probability $\left(\mathrm{CP}_{i j}\right)$ for a packet carrier to meet at least one contact toward road $r_{i j}$ when the carrier moves within the intersection area. Let $\mathcal{N}\left(T_{i j}\right)$ denote how many contacts moving toward road $r_{i j}$ can be seen in the intersection area within time interval $T_{i j}$, and let $\lambda_{i j}$ denote the average rate of contacts leaving $I_{i}$ and moving toward road $r_{i j}$, which can be computed as $\lambda_{i j}=\rho_{i j} \cdot v_{i j}\left(\rho_{i j}\right.$ and $v_{i j}$ are defined in Section II-C). According to the definition of Poisson distribution

$$
\begin{aligned}
\mathrm{CP}_{i j} & =\operatorname{Prob}\left(\mathcal{N}\left(T_{i j}\right) \geq 1\right) \\
& =1-\operatorname{Prob}\left(\mathcal{N}\left(T_{i j}\right)=0\right) \\
& =1-e^{-\lambda_{i j} T_{i j}} \frac{\left(\lambda_{i j} T_{i j}\right)^{0}}{0 !} \\
& =1-e^{-\rho_{i j} v_{i j} T_{i j}} .
\end{aligned}
$$

The VADD protocols forward a packet toward the best possible direction at the intersection. If intersection $I_{i}$ only has two outgoing roads $r_{i a}$ and $r_{i b}$ and satisfies $D_{i a}<D_{i b}$ with contacting probability $\mathrm{CP}_{i a}$ for contacts toward road $r_{i a}$ and $\mathrm{CP}_{i b}$ for contacts toward road $r_{i b}$, respectively, $P_{i a}$ would be equal to $\mathrm{CP}_{i a}$, and $P_{i b}$ would be $\mathrm{CP}_{i b}-\mathrm{CP}_{i a} \cdot \mathrm{CP}_{i b}$. This is due to the reason that the path with the expected minimum delivery delay will be selected if both contacts are available when the packet carrier passes intersection $I_{i}$. Therefore, to compute $P_{i j}$ at $I_{i}$, we need to first sort $\mathrm{CP}_{i j}$ for all $j \in N(i)$ by the nondecreasing order of $D_{i j}$. However, as $D_{i j}$ cannot be obtained at this stage, we use the angle between the direction of road $r_{i j}$ and the vector from the current intersection to the destination, which is denoted as $\theta_{i j}$, to approximate $D_{i j}$, because a road with a 
smaller angle will more likely lead to a location closer to the destination. The sorted list of $\mathrm{CP}_{i j}$ looks like

$$
\mathrm{CP}_{i j_{1}}, \mathrm{CP}_{i j_{2}}, \mathrm{CP}_{i j_{3}}, \ldots, \mathrm{CP}_{i j_{n}}, \quad \text { where } n=|N(i)| \text {. }
$$

The subscripts of $j_{i}$ s implicitly indicate a meaningful order

$$
\theta_{i j_{1}} \leq \theta_{i j_{2}} \leq \theta_{i j_{3}} \leq \cdots \leq \theta_{i j_{n}}
$$

By using basic probability, we can calculate the probability of a packet being forwarded to road $r_{i j}$ at $I_{i}$. This result is denoted as $P_{i j}^{\prime}$

$$
\begin{aligned}
P_{i j_{1}}^{\prime}= & \mathrm{CP}_{i j_{1}} \\
P_{i j_{2}}^{\prime}= & \mathrm{CP}_{i j_{2}}-\mathrm{CP}_{i j_{1}} \cdot \mathrm{CP}_{i j_{2}} \\
P_{i j_{3}}^{\prime}= & \mathrm{CP}_{i j_{3}}-\left(\mathrm{CP}_{i j_{1}} \cdot \mathrm{CP}_{i j_{3}}+\mathrm{CP}_{i j_{2}} \cdot \mathrm{CP}_{i j_{3}}\right) \\
& +\mathrm{CP}_{i j_{1}} \cdot \mathrm{CP}_{i j_{2}} \cdot \mathrm{CP}_{i j_{3}}
\end{aligned}
$$

Supposing that the packet carrier will move to road $r_{i j_{c}}$ (either go straight or make a turn) after passing $I_{i}$, the packet will only be forwarded to the road that has higher or equal priority. That is, for a road $r_{i j_{k}}$, if $k>c, P_{i j_{k}}$ is equal to zero since the carrier will continue to buffer data instead of forwarding it toward lower priority roads. Thus, under the condition that the packet carrier goes to road $r_{i j_{c}}$ after leaving $I_{i}$, the probability that road $r_{i j_{p}}$ will be chosen as the packet-forwarding direction can be defined as the following conditional probability:

$$
P_{i j_{p} \mid i j_{c}}=\operatorname{Prob}\left\{\text { packet forwarded to } r_{i j_{p}} \mid \text { carrier goes to } r_{i j_{c}}\right\}
$$

and

$$
P_{i j_{p} \mid i j_{c}}= \begin{cases}P_{i j_{p}}^{\prime}, & \forall p<c \\ 1-\sum_{s=1}^{c-1} P_{i j_{s}}^{\prime}, & p=c \\ 0, & \forall p>c .\end{cases}
$$

Let $Q_{i c}$ denote the probability of a vehicle moving (going straight or turning) from the current intersection $I_{i}$ toward the next adjacent intersection $I_{c} . P_{i j}$ can be calculated by the following:

$$
P_{i j}=\sum_{c \in N(i)} Q_{i c} \times P_{i j_{p} \mid i j_{c}} .
$$

The complexity of calculating $P_{i j}$ is dominated by the step of calculating $P_{i j}^{\prime}$, and it is given by

$$
\Theta\left(\sum_{k=1}^{N(i)}\left(\begin{array}{c}
N(i) \\
k
\end{array}\right)\right)=\Theta\left(2^{N(i)}\right) .
$$

Since one intersection is only directly connected with several neighboring intersections in reality, $N(i)$ is bounded and fairly small; therefore, $2^{N(i)}$ can be seen as a constant. Therefore, the complexity of computing $P_{i j}$ for all $n$ roads inside the boundary is $\Theta(n)$.

\section{Data Forwarding in Straightway and Destination Modes}

Data forwarding in the StraightWay Mode is much simpler than the Intersection Mode since the traffic is at most bidirectional. We can simply specify the intersection ahead, which is joined by the current road, as the target and then apply GPSR [16] toward the target location. If there is no vehicle available to forward ahead, the current packet carrier continues to carry the packet. Certainly, there may be better solutions. For example, when the packet carrier meets a vehicle in the opposite direction, the estimated delay from the current vehicle position may be different when the vehicle received the packet. As a result, the packet carrier may decide to take the intersection behind as the target location. However, checking such cases may increase the computation overhead, and the chance of such cases may be small. Due to space limit, we will leave these optimizations as future work.

A packet switches to the Destination Mode when its distance to the destination is below a predefined threshold. The location of the destination becomes the target location, and GPSR is used to deliver the packet to the final destination.

\section{Performance Evaluations}

In this section, we evaluate the performance of three VADD protocols, namely, L-VADD, D-VADD, and H-VADD. Since the L-VADD protocol may have routing loops, we evaluate two versions of them: L-VADD (with loop) and L-VADD (loop-free). It is shown in our simulation that almost all the intersection routing loops in L-VADD (with loop) can be detected by checking the previous three-hop information so that L-VADD (loop-free) encloses the previous three-hop information in every forwarding packet to avoid intersection routing loops. The H-VADD protocol is a hybrid version of the L-VADD protocol and the D-VADD protocol. We compare the performance of the VADD protocols to several existing protocols: dynamic source routing (DSR) protocol [18], the epidemic routing protocol [12], and GPSR [16]. Since GPSR is not proposed for sparsely connected networks, its performance is very poor in VANETs. To have a fair comparison, we extend GPSR by adding buffers. In this way, GPSR (with buffer) can be considered as a simple carry and forward protocol.

The experiment is based on a $4000 \times 3200 \mathrm{~m}$ rectangle street area, which presents a grid layout. The street layout is derived and normalized from a snapshot of a real street map in Topologically Integrated Geographic Encoding and Referencing (TIGER) database [19] from the U.S. Census Bureau. These map data are transformed into a data format that can be used by ns2, based on techniques presented in [20]. The MAC layer protocol follows 802.11, with the distributed coordination function enabled.

The mobility pattern is generated similar to that of [20], but we need to unevenly model the distributed traffic. We revised the software in [20] to first compute the traveling time on each 


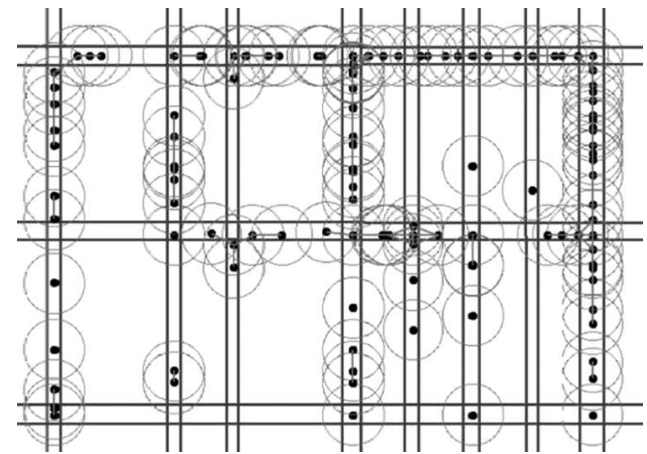

Fig. 8. Snapshot of the simulation setup area.

TABLE I

SIMULATION SETUP

\begin{tabular}{|l|c|}
\hline Parameter & Value \\
\hline Simulation area & $4000 \mathrm{~m} \times 3200 \mathrm{~m}$ \\
\hline \# of intersections & 24 \\
\hline Intersection area radius & $200 \mathrm{~m}$ \\
\hline Number of vehicles & 150,210 \\
\hline \# of packet senders & 15 \\
\hline Communication range & $15-80$ miles per hour \\
\hline Vehicle velocity & $0.1-1$ packet per second \\
\hline CBR rate & $10 \mathrm{~B}-4 \mathrm{~KB}$ \\
\hline Data packet size & $0.5 \mathrm{sec}$ \\
\hline Vehicle beacon interval & $128 \mathrm{sec}$ \\
\hline Packet TTL & \\
\hline
\end{tabular}

road based on the length and speed limit of the road and then let each vehicle select the shortest path to the destination. Thus, roads with high speed limit are chosen with higher probability, which generates uneven traffic density. The initial distribution follows the traffic density distribution of the original map (i.e., more crowded roads are deployed with relatively more vehicles and less interspace between vehicles). Then, each vehicle randomly chooses one of the intersections as its destination and moves along the road to this destination. Immediately after it arrives in the destination, the vehicle randomly selects another intersection as the next destination and moves toward it. The TIGER database contains road-type information for each road, and we assign the speed limit $(20-75 \mathrm{mi} / \mathrm{h})$ to each road based on the road-type information, for example, $20 \mathrm{mi} / \mathrm{h}$ for unseparated downtown streets and $75 \mathrm{mi} / \mathrm{h}$ for highways. The vehicles follow the speed limit assigned to the road they are traveling on, with a variance of $5 \mathrm{mi} / \mathrm{h}$. For simplicity, we only consider the case of isolated intersection, and the node contacting time at an intersection is calculated by (5). Fig. 8 shows a snapshot of the simulation area.

Two fixed sites are deployed on the rightmost vertical road in Fig. 8. Among all vehicles, 15 of them are randomly chosen to send constant bit rate (CBR) data packet to one of the fixed sites during the move. To evaluate the performance on different data transmission density, we vary the data-sending rate (CBR rate) from 0.1 to 1 packet/s. All experiment parameters are shown in Table I. In order to find out the direction where a packet is forwarded to a given fixed site, priority ranking of the outgoing roads at the intersections for that fixed site is precomputed and loaded to the vehicle as the simulation starts. The performance of the protocols is measured by using the data-delivery ratio, the data-delivery delay, and the generated traffic overhead.

\section{A. Data-Delivery Ratio}

In this section, we compare the performance of VADD protocols with epidemic routing, GPSR (with buffer), and DSR in terms of data-delivery ratio and examine how it is affected by the data transmission and vehicle densities.

Fig. 9 shows the data-delivery ratio as a function of the datasending rate and compares the performance under different vehicle density settings. As shown in the figure, DSR has the lowest data-delivery ratio and is not suitable for sparsely connected vehicular networks. Although GPSR (with buffer) is implemented in a carry and forward way, it is not a good choice since the geographical approach sometimes leads to void areas with few vehicles passing by, and it cannot make use of the traffic patterns. Therefore, its delivery ratio is poor when the vehicle density is low, as shown in Fig. 9(a). However, when vehicle density is high [Fig. 9(b)], where the connectivity is much better than the previous scenario, GPSR achieves a very good delivery ratio since the node mobility will help carry and forward the packets which temporarily reach the void zone. Intuitively, epidemic routing explores every possible path to the destination and should represent the upper bound of the data-delivery ratio. This is true when the datasending rate is low (e.g., when the data rate is 0.1 packet/s) and the node density is low. However, as the data-sending rate increases, the epidemic routing protocol underperforms most VADD protocols. This is due to MAC layer collisions. As the number of data requests increases, the network traffic dramatically increases in epidemic routing (see Fig. 12), thus increasing the number of collisions and reducing the packetdelivery ratio. At more densely deployed network [Fig. 9(b)], the delivery ratio of the epidemic protocol drops even faster. While epidemic routing is very sensitive to the data rate and nodes density, the VADD protocols, particularly H-VADD, steadily hold the close-to-optimum delivery ratio at different settings.

Fig. 9 also compares several VADD protocols. Among them, the H-VADD protocol has the benefits of both L-VADD and D-VADD, presenting the best delivery ratio. As discussed in the previous section, loop detection prevents some packets from being sent to the loop vulnerable neighbors, which reduces the chance of using some valid good paths. However, with a high vehicle density, intersection routing loops do not frequently occur, and the L-VADD (loop-free) protocol does not need to exclude too many innocent nodes to recover from the loop; its delivery ratio becomes higher.

The L-VADD (with loop) protocol has the lowest datadelivery ratio among the VADD protocols and performs particularly poor when the node density is low since routing loops frequently happen and lead to packet drops. Fig. 10 compares the percentage of the data packet dropped due to TTL or MAC layer collision at a 150-node setting. As shown in the figure, three VADD protocols (L-VADD, D-VADD, and H-VADD) have similar percentage of packet drops. Compared to these VADD protocols, the L-VADD (with loop) protocol has a much higher packet drop rate, i.e., about five times higher. Fig. 10 also verifies the effectiveness of the routing loop detection mechanism used by the loop-free L-VADD protocol. 


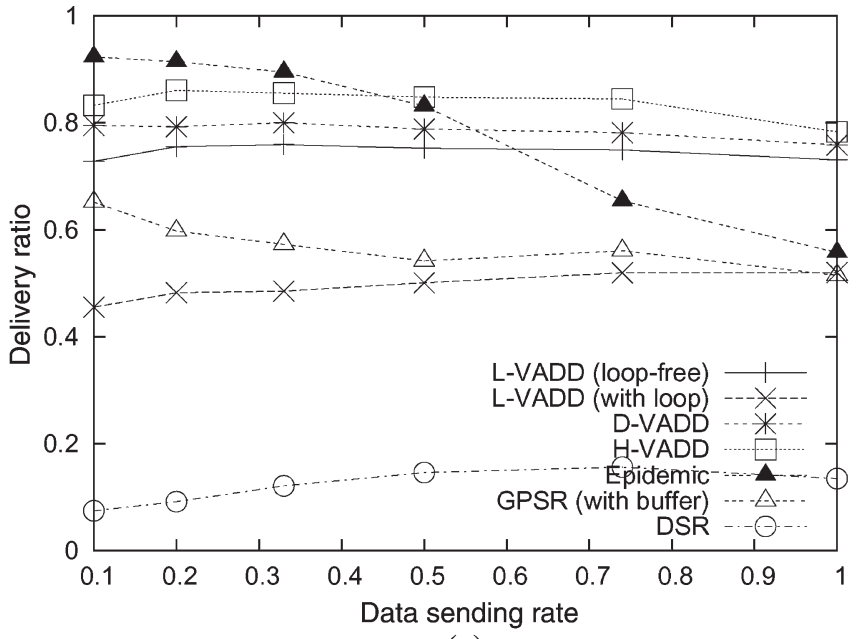

(a)

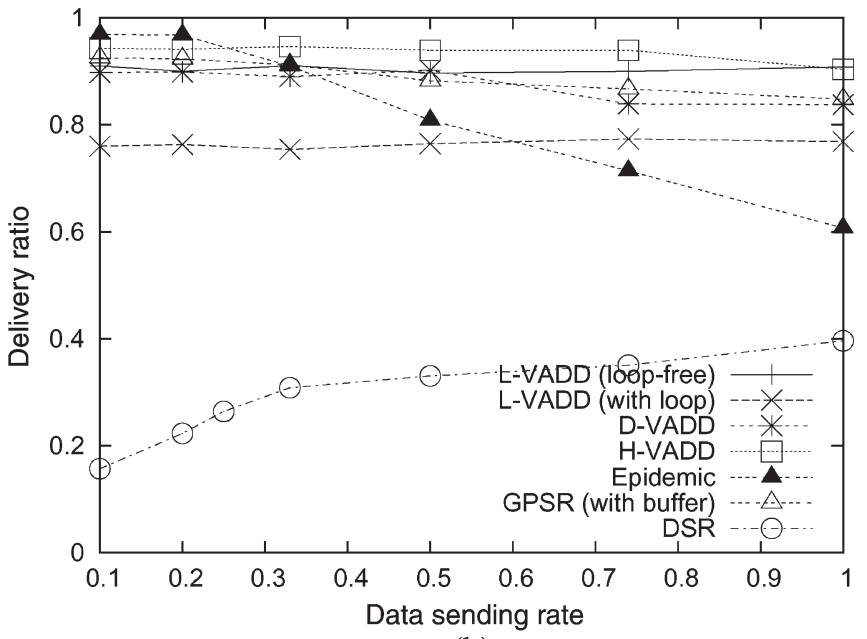

(b)

Fig. 9. Data-delivery ratio as a function of the data-sending rate. (a) 150 nodes. (b) 210 nodes.

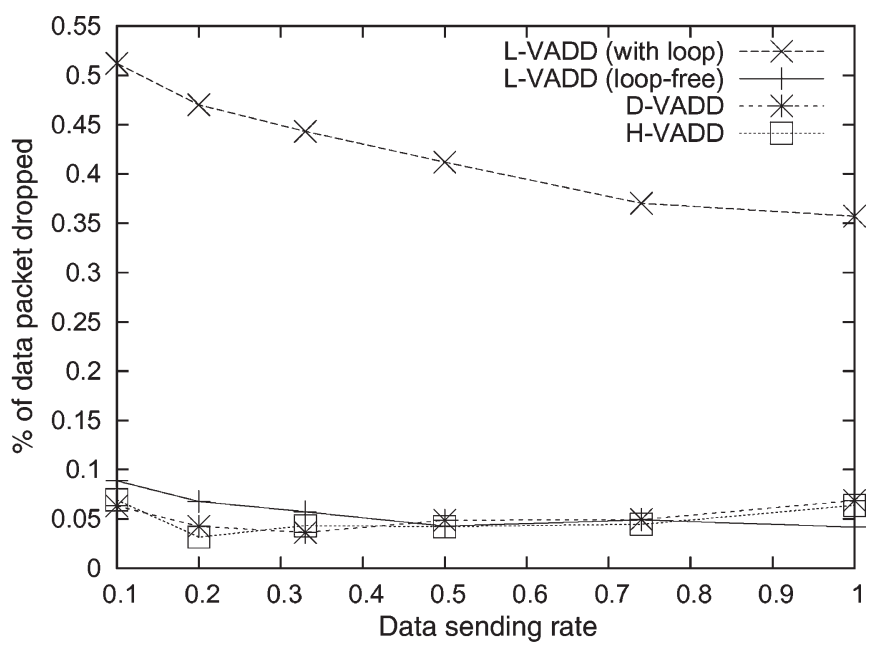

Fig. 10. Percent of data packets dropped due to routing loops or MAC layer packet collisions (150 nodes).

From the figure, we can also see that the dropping rate of the L-VADD (with loop) protocol is reduced as the data-sending rate increases. This is because most packets are dropped due to routing loops, instead of congestion using the 150-node setting. Routing loops only occur at some particular time intervals. When the data-sending rate is high, more packets are buffered and delivered before a routing loop occurs. Since the number of dropped packets due to routing loops does not change too much, but the total number of delivered packets increases as the data-sending rate increases, the percentage of data packet drops become lower when the data-sending rate increases.

\section{B. Data-Delivery Delay}

In this section, we compare the data-delivery delay from moving vehicles to fixed sites using carry and forward schemes. Here, we do not consider DSR since its data-delivery ratio is too low. Similarly, we do not consider the L-VADD protocol due to its low delivery ratio compared to the D-VADD protocol. Note that a low delivery ratio may reduce the average data- delivery delay since most undelivered packets may experience long delay. This is particularly true in the DSR protocol, which only forwards packets through wireless communication, whereas other carry and forward protocols also rely on vehicle movement.

Fig. 11 shows the change of the data-delivery delay by increasing the data-sending rate. Epidemic routing presents the optimum delivery delay only when the data rate is very low. As the data-sending rate increases, the delay of the epidemic routing scheme also increases because epidemic routing generates many redundant packets. As the traffic load increases, many packets may be dropped. Even though the redundant copies can help deliver the packet, the delay increases. GPSR has a relatively low data-delivery delay at low node density [Fig. 11(a)], but it is not meaningful simply because of its low delivery ratio. A valid comparison is when the GPSR protocol, the epidemic routing protocol, and the VADD protocols have similar delivery ratio, e.g., at a data rate below 0.4 in Fig. 11(b). In this case, GPSR shows a much longer delivery delay because it does not consider the vehicle traffic pattern when making decisions.

The H-VADD protocol presents similar delivery delay as the D-VADD protocol when the vehicle density is low since it relies more on D-VADD for loop recovery because of more routing loops. When the vehicle density is high, the delay of the $\mathrm{H}-\mathrm{VADD}$ protocol is lower than that of the D-VADD protocol but close to that of the L-VADD protocol. This shows that it behaves more like the L-VADD protocol but has a better packet-delivery ratio than the loop-free L-VADD. These results verify that H-VADD effectively captures the advantages of both L-VADD and D-VADD.

The delivery delay is affected by the delivery ratio. Some extreme long-delay packets may greatly increase the mean value, and the average delivery delay generally becomes smaller when a few packets are successfully delivered. Therefore, the delivery delay of H-VADD appears to be larger than some other VADDs simply because it delivers more packets. To better study the delivery delay, we examine the "the lowest $75 \%$ delivery delay," which is the average delay of the lowest $75 \%$ packets. As shown 


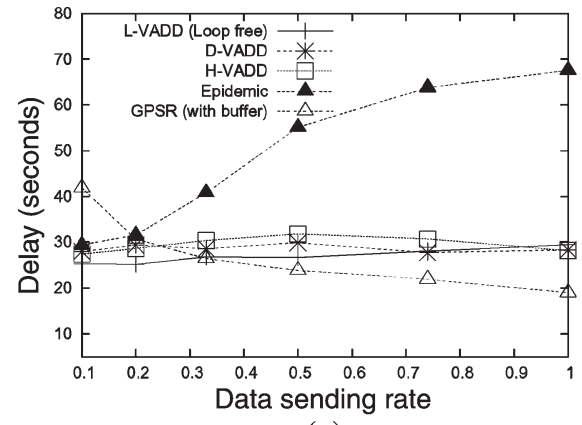

(a)

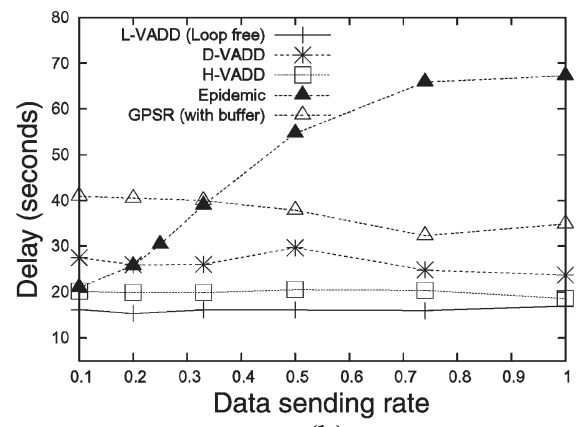

(b)

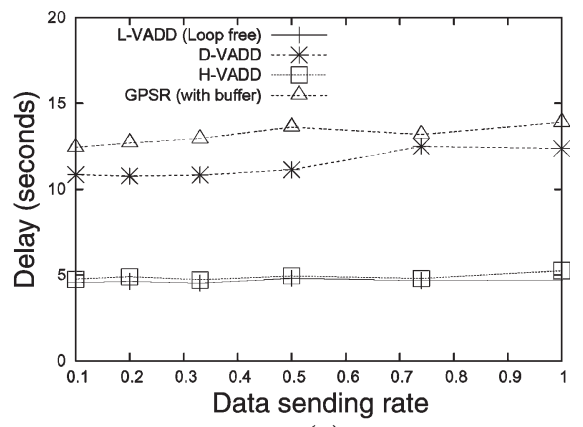

(c)

Fig. 11. Data-delivery delay as a function of the data-sending rate. (a) 150 nodes. (b) 210 nodes. (c) Lowest $75 \%$ delivery delay (210 nodes).

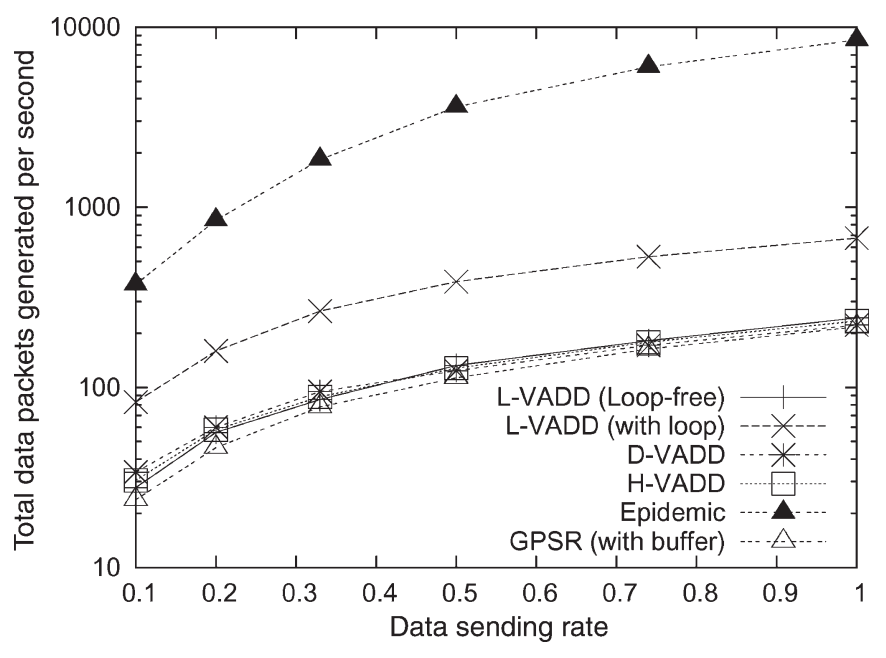

Fig. 12. Number of packets generated.

in Fig. 11(c), the delay of H-VADD is only half of D-VADD. It is similar to L-VADD because it behaves more like L-VADD when the node density is high.

\section{Data Traffic Overhead}

In this section, we evaluate the overhead of the carry and forward protocols by using the number of data packets generated per second, which is a summation of individual packet hops. For example, if a generated packet is forwarded ten hops, the packet overhead is counted as ten packet hops. The control packets are not included. The reason is that the proposed VADD protocol is essentially a location-based routing protocol, and it does not require any more control packets than other locationbased routing protocols. All VADD protocols and GPSR require the same number of control messages, which are the beacon messages that are used to report the node location. The control message overhead depends on the beacon interval, which is set to $0.5 \mathrm{~s}$ for all the evaluated protocols. Thus, in VADD protocols and GPSR, each node generates the same amount of control traffic, regardless of the data rate, topology, and mobility. All results shown in this section are based on the 210-node deployment scenario. Fig. 12 shows the generated packet overhead as a function of the data-sending rate. As the data-sending rate increases, the number of packets generated by all protocols also increases. However, the increasing trend is different. The overhead of epidemic routing increases much faster than other protocols due to the redundant packets generated.

For the VADD protocols, L-VADD (with loop) has the highest overhead due to loops, whereas all the other VADD protocols have about the same low overhead compared to D-VADD.

\section{Impact of Data Packet Size}

Fig. 13 shows the impact of data packet size on the performance of the GPSR protocol, the epidemic routing proto$\mathrm{col}$, and the VADD protocols. Since all the VADD protocols are affected by the data size in a similar way, we choose H-VADD in representing the VADD protocols in the comparison. Larger packet size consumes more bandwidth and generates more contention for the limited wireless channel. As shown in Fig. 13(a), the total injected data traffic using the epidemic protocol increases much faster than GPSR and H-VADD. We intentionally choose the setting at a very low data-sending rate $(0.1 / \mathrm{s})$, where the delay of the epidemic routing is close to H-VADD, and the delivery ratio is slightly better than H-VADD at the starting size $(10 \mathrm{~B})$ due to the help of a large amount of redundant packets. The delivery ratio of the epidemic routing protocol drops much faster than the H-VADD protocol as the data size increases [see Fig. 13(b)]. As shown in Fig. 13(c), the delivery delay of the epidemic protocol dramatically increases as the packet size increases due to the congestion caused by the huge traffic load. The delay of the GPSR protocol slightly decreases as the packet size increases since some long delay packets are dropped. From the figure, we can also see that the H-VADD protocol has the lowest datadelivery delay for different data sizes.

\section{CONCLUSion AND Future Work}

Many researchers and industry players believe that the benefit of vehicular networks on traffic safety and many commercial applications [1] should be able to justify the cost. With such a vehicular network, many data-delivery applications can be supported without extra hardware cost. However, existing protocols are not suitable in supporting delay-tolerant applications in sparsely connected vehicular networks. To address this problem, we adopted the idea of carry and forward, where a moving vehicle carries the packet until a new vehicle moves into its vicinity and forwards the packet. Being different from existing carry and forward solutions, we 


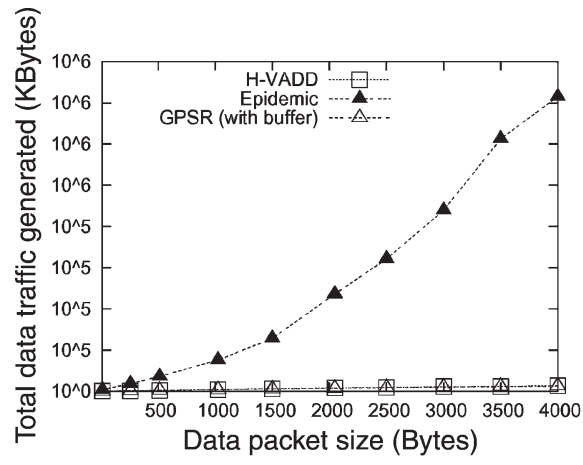

(a)

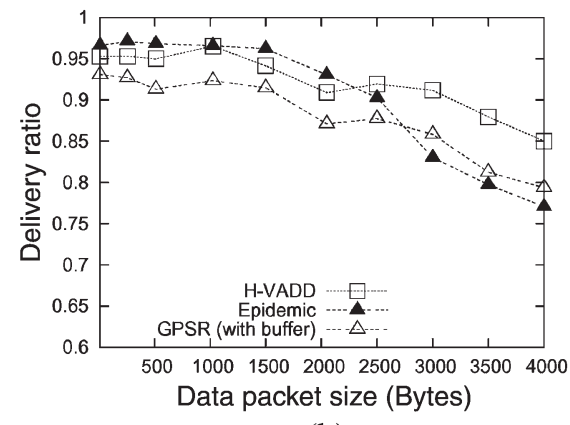

(b)

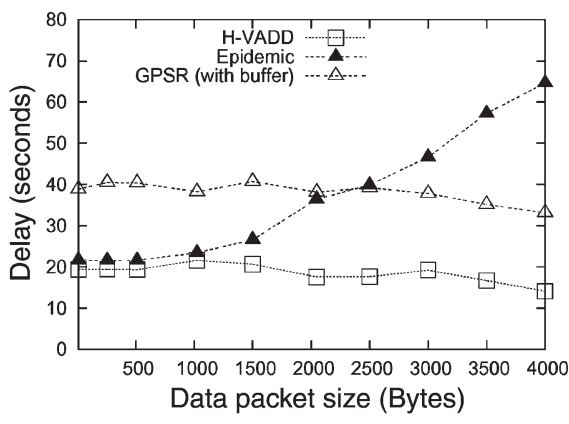

(c)

Fig. 13. Impact of data packet size. (a) Total amount of data traffic generated. (b) Data-delivery ratio. (c) Data-delivery delay.

make use of the predictable vehicle mobility, which is limited by traffic pattern and road layout. We proposed several VADD protocols, namely, L-VADD, D-VADD, and H-VADD, based on the techniques used for road selection at the intersection. Experimental results showed that the proposed VADD protocols outperform existing solutions in terms of packet-delivery ratio, data packet delay, and traffic overhead. Among the proposed VADD protocols, the H-VADD protocol has a much better performance.

As future work, we will design protocols for query data return. This is different from the previous data-delivery protocol since the destination is moving. Simple solutions can be based on the predictable vehicle mobility. By adding the moving trajectory into the query packet, the information server attaches the moving trajectory with the query reply. Intermediate vehicles that deliver the query reply need to calculate the destination position and deliver the query reply to that position. We will design and evaluate such protocols and investigate other better solutions. In addition, caching techniques [21], [22] may also be applied to VANET to reduce the query delay.

\section{APPENDIX}

\section{Proof of THE Linear EQUATION SYSTEM}

Theorem 2: The linear equation system given by (4) has a unique solution.

In (4), if $P-E$ is an $n \times n$ invertible matrix, $(P-E)$. $X=-D$ has a unique solution given by $X=(P-E)^{-1}$. $-D$. The rest of this section will prove that matrix $P-E$ used in Section II-C is invertible.

It is important to relate matrix $P-E$ to real road networks to further illustrate the properties of $P-E$. Matrix $E$ is simply an $n \times n$ identity matrix. The $n \times n$ matrix $P$ describes the system with $n$ directional roads. Note that one road with two opposite traffics is defined as two different directional roads in our model. Each row of $P$ represents a directional road, and each column represents a directional road. Most importantly, the number in the $i$ th row and $j$ th column of $P$ (called the $i j$ th element and written as $P_{i j}$ ) represents the probability of choosing road $j$ as the next road to forward a packet, given that the packet is currently on road $i$. Let $p_{i j}$ denote the $i j$ th element in matrix $P-E$. The following three properties of $P-E$ are useful in proving Theorem 2.

\section{Property 1-Diagonal Property:}

$$
p_{k k}=-1, \quad \text { for each } k=1, \ldots, n .
$$

Proof: If a packet is currently carried on road $k$, the next road to forward the packet cannot be itself. Therefore, the probability of selecting itself as the next road is zero. Therefore, in matrix $P, P_{k k}=0$, for each $k=1, \ldots, n$. The values of the diagonal elements in $P-E$ are

$$
p_{k k}=P_{k k}-1=0-1=-1, \quad \text { for each } k=1, \ldots, n .
$$

Property 2-Row Property: There exists at least one row $r$ in $P-E$, such that $p_{r k}=0$, for each $k=1, \ldots, n$ and $k \neq r$. Besides these rows, all the other rows $r^{\prime}$ satisfy $\sum_{k=1, k \neq r^{\prime}}^{n} p_{r^{\prime} k}=1$.

Proof: Let us first examine matrix $P$. Since we assume that the destination area is either within one intersection area or at the middle of the road connecting two intersections, we can find at least one road which directly leads to the destination (without using any intermediate intersections). Let us call this road $r$. When a packet is already carried on road $r$, it will not be forwarded to any other road except the destination. Thus, the probability of the packet to reach any other road from road $r$ is zero, i.e., $P_{r k}=0$, for each $k=1, \ldots, n$ and $k \neq r$. When the packet is on a road which does not directly lead to the destination (named $r^{\prime}$ ), it may be forwarded to any of the roads directly connected with the current road with certain probability, and the summation of the probabilities of being forwarded to all these roads is $\sum_{k=1, k \neq r^{\prime}}^{n} P_{r^{\prime} k}=1$.

Apparently, $P$ and $P-E$ have exactly the same elements, except the diagonal elements. Therefore, the aforementioned properties are also used for matrix $P-E$. The row property of $P-E$ is proved.

Property 3-Column Property: At any column $c$ of matrix $P-E$, the element $p_{k c}$ is either zero or a positive value less than or equal to one, for each $k=1, \ldots, n$ and $k \neq c$.

Proof: In matrix $P$, the value of the element $P_{k c}$ describes the probability of road $c$ to be chosen as the next road to forward the packet, when the packet is currently on road $k$. When road $c$ is not directly connected to road $k$, it is impossible for road $c$ to be the next road to forward the packet after road $k$, so $P_{k c}$ is equal to zero. Otherwise, the packet may be forwarded to 
road $c$ immediately after passing road $k$, and the probability is apparently a positive value less than or equal to one.

Again, since $P$ and $P-E$ have exactly the same elements except for the diagonal elements, $p_{k c}$ is equal to $P_{k c}$, which is either zero or a positive value less than or equal to one, when $k \neq c$.

Let us first simplify (4) by eliminating all the equations with the form

$$
-x_{i}=-d_{i}
$$

The equation of this form corresponds to one row vector $P_{i}$ in $P$, with $p_{i j}=0(j=1, \ldots, n)$, which represents the road directly leading to the destination. We simply substitute all $x_{i}$ for $d_{i}$ in these equations in $P-E$ and call the simplified new $m \times m$ (certainly $m<n$ ) matrix as $A$. Apparently, $A$ still holds the aforementioned three properties of $P-E$ because this simple transformation does not change any of the aforementioned properties. In addition, since $A$ is reduced from $P-E$ only by using elementary row operations, proving $A$ to be invertible is equivalent to proving $P-E$ to be invertible.

A sufficient condition that will guarantee a matrix to be invertible is that this matrix is diagonally dominant and irreducible.

Definition 1: A matrix $Q_{m \times m}$ is said to be diagonally dominant iff, for every row (or column), the sum of the absolute values of the off diagonal elements is never greater than the absolute value of the diagonal element, and at least there is one row $i$ in $Q$ such that

$$
\left|q_{i i}\right|>\sum_{\substack{k=1 \\ k \neq i}}^{m}\left|q_{i k}\right| .
$$

Definition 2: A matrix $Q_{m \times m}$ is said to be irreducible iff, for any row index $i$ and column index $j$, there is always a nonnegative integer $s$ (which may be zero) and a sequence of integers $k_{1}, \ldots, k_{s}$ so that the product

$$
q_{i, k_{1}} \times q_{k_{1}, k_{2}} \times \cdots \times q_{k_{s}, j}
$$

is nonzero.

Lemma 1: Matrix $A$ is a diagonally dominant matrix.

Proof: Since Properties 1-3 are held in $A$, all the values of the diagonal elements in $A$ are equal to $0-1=-1$ (Property 1), and the sum of the absolute values of the off diagonal elements is less than or equal to one (Property 2). Furthermore, the transformation from matrix $P-E$ to matrix $A$ eliminates some columns, and the eliminated columns represent the roads which directly lead to the destination. For simplicity, supposing that only one column $j$ is eliminated in $P-E$, thus, road $j$ is the only road directly leading to the destination. Since there must exist at least one other road $i$ (assume $i<j$, without loss of generality), which does not directly lead to the destination, choose road $j$ with certain probability $P_{i j}\left(P_{i j} \neq 0\right)$ as the next road to forward the packet (otherwise, the packet cannot reach the destination when it is on road $i$ ). Since $P_{i j}$ is equal to the element $p_{i j}$ in matrix $P-E$, when column $j$ in $P-E$ is eliminated, the sum of the absolute values of the off diagonal elements in row $i$ is reduced and becomes less than one. Therefore, we find one row $i$ in the new $(n-1) \times(n-1)$ matrix $A$, satisfying

$$
\left|a_{i i}\right|=1>\sum_{\substack{k=1 \\ k \neq i}}^{n-1}\left|a_{i k}\right| .
$$

When more than one columns are eliminated, this property can similarly be proved. Therefore, matrix $A$ is diagonally dominant.

Lemma 2: Matrix $A$ is an irreducible matrix.

Proof: Since $P-E$ is generated based on the real roads in a given nonpartitioned area, all the roads are reachable from one to another. Thus, for any two road $i$ and $j$, a packet can always be routed from $i$ to $j$ with certain probability. The only exception occurs when the packet is already on the road directly leading to the destination, and it is impossible to reach any other road. However, after we eliminate these roads in $P-E$ and transform the matrix to $A$, this exception does not exist in $A$ because all the roads directly leading to the destination are eliminated. Therefore, the probability of the packet routed between any pair of roads $i$ and $j$ is not zero. Suppose that the packet is routed via the road sequence $i, r_{k_{1}}, r_{k_{2}}, \ldots, r_{k_{s}}, j$. The probability of following this sequence is

$$
a_{i, k_{1}} \times a_{k_{1}, k_{2}} \times \cdots \times a_{k_{s}, j}
$$

which is not zero. Thus, matrix $A$ is irreducible.

Since matrix $A$ is both diagonally dominant and irreducible, it is invertible. We conclude that matrix $P-E$ is also invertible, and the linear equation system shown in (4) has a unique solution.

\section{REFERENCES}

[1] X. Yang, J. Liu, F. Zhao, and N. Vaidya, "A vehicle-to-vehicle communication protocol for cooperative collision warning," in Proc. Int. Conf. MobiQuitous, Aug. 2004, pp. 114-123.

[2] J. Yin, T. Eibatt, G. Yeung, B. Ryu, S. Habermas, H. Krishnan, and T. Talty, "Performance evaluation of safety applications over DSRC vehicular ad hoc networks," in Proc. VANET, Oct. 2004, pp. 1-9.

[3] R. Verdone, "Multi-hop R-Aloha for inter-vehicle communication at millimeter waves," IEEE Trans. Veh. Technol., vol. 46, no. 4, pp. 992-1005, Nov. 1997.

[4] M. Lott, R. Halmann, E. Schulz, and M. Radimirsch, "Medium access and radio resource management for ad hoc networks based on UTRA TDD," in Proc. ACM Mobihoc-Poster, 2001, pp. 76-86.

[5] Q. Xu, T. Mark, J. Ko, and R. Sengupta, "Vehicle-to-vehicle safety messaging in DSRC," in Proc. VANET, Oct. 2004, pp. 19-28.

[6] G. Korkmaz, E. Ekici, F. Ozguner, and U. Ozguner, "Urban multi-hop broadcast protocol for inter-vehicle communication systems," in Proc. VANET, Oct. 2004, pp. 76-85.

[7] B. Xu, A. Ouksel, and O. Woflson, "Opportunistic resource exchange in inter-vehicle ad hoc networks," in Proc. IEEE Int. Conf. MDM, 2004, pp. 4-12.

[8] S. Ghandeharizadeh, S. Kapadia, and B. Krishnamachari, "PAVAN: A policy framework for content availability in vehicular ad-hoc networks," in Proc. VANET, Oct. 2004, pp. 57-65.

[9] R. Frenkiel, B. Badrinath, J. Borras, and R. Yates, "The infostations challenge: Balancing cost and ubiquity in delivering wireless data," IEEE Pers. Commun., vol. 7, no. 2, pp. 66-71, Apr. 2000.

[10] D. Goodman, J. Borras, N. Mandayam, and R. Yates, "INFOSTATIONS: A new system model for data and messaging services," in Proc. IEEE VTC, Rome, Italy, May 1997, vol. 2, pp. 969-973. 
[11] V. Namboodiri, M. Agarwal, and L. Gao, "A study on the feasibility of mobile gateways for vehicular ad-hoc networks," in Proc. VANET, Oct. 2004, pp. 66-75.

[12] A. Vahdat and D. Becker, "Epidemic routing for partially connected ad hoc networks," Duke Univ., Durham, NC, Tech. Rep. CS-200006, 2000.

[13] W. Zhao, M. Ammar, and E. Zegura, "New directions: A message ferrying approach for data delivery in sparse mobile ad hoc networks," in Proc. ACM MobiHoc, 2004, pp. 187-198.

[14] Q. Li and D. Rus, "Sending messages to mobile users in disconnected ad-hoc wireless networks," in Proc. ACM Mobicom, 2000, pp. 44-55.

[15] GB Traffic Volumes. (2005, May). [Online]. Available: www. mapmechanics.com

[16] B. Karp and H. T. Kung, "GPSR: Greedy perimeter stateless routing for wireless networks," in Proc. ACM MOBICOM, Aug. 2000, pp. 243-254

[17] J. Blum, A. Eskandarian, and L. Hoffman, "Challenges of intervehicle ad hoc networks," IEEE Trans. Intell. Transp. Syst., vol. 5, no. 4, pp. 347351, Dec. 2004.

[18] D. Johnson and D. Maltz, "Dynamic source routing in ad hoc wireless networks," in Mobile Computing. Norwell, MA: Kluwer, 1996, pp. 153-181.

[19] Tiger, Tiger/Line and Tiger-Related Products, U.S. Census Bureau. [Online]. Available: http://www.census.gov/geo/www/tiger/

[20] A. K. Saha and D. B. Johnson, "Modeling mobility for vehicular ad hoc networks," in Proc. VANET-Poster, Philadelphia, PA, Oct. 2004, pp. 91-92.

[21] L. Yin and G. Cao, "Supporting cooperative caching in ad hoc networks," IEEE Trans. Mobile Comput., vol. 5, no. 1, pp. 77-89, Jan. 2006.

[22] G. Cao, "A scalable low-latency cache invalidation strategy for mobile environments," IEEE Trans. Knowl. Data Eng., vol. 15, no. 5, pp. 12511265, Sep./Oct. 2003.

[23] J. Zhao and G. Cao, "VADD: Vehicle-assisted data delivery in vehicular ad hoc networks," in Proc. IEEE INFOCOM, Apr. 2006, pp. 1-12.

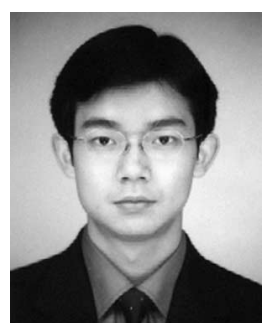

Jing Zhao (S'06) received the B.S. degree from Peking University, Beijing, China. He is currently working toward the Ph.D. degree in computer science and engineering with the Department of Computer Science and Engineering, The Pennsylvania State University, University Park.

His research interests include distributed systems, wireless networks, and mobile computing, with a focus on mobile ad hoc networks.

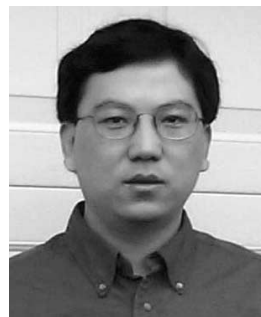

Guohong Cao (S'98-A'99-M'03-SM'07) received the B.S. degree from Xi'an Jiaotong University, Xi' an, China, and the M.S. and Ph.D. degrees in computer science from The Ohio State University, Columbus, in 1997 and 1999, respectively.

$\mathrm{He}$ is currently an Associate Professor with the Department of Computer Science and Engineering, The Pennsylvania State University, University Park. His research interests include wireless networks and mobile computing. He has published over 100 papers in the areas of sensor networks, wireless network security, data dissemination, resource management, and distributed fault-tolerant computing.

Dr. Cao is an Editor of the IEEE TRANSACTIONS ON Mobile COMPUTING and the IEEE TRANSACTIONS ON WiRELESS COMMUNICATIONS and has served on the program committee of many conferences. He was a recipient of the NSF CAREER award in 2001. 